\title{
Studies of Magnetoresistance in GaAs:Te Crystals with Structural Disorder at Doping Limit
}

\author{
T. TARKOWski, T. SŁupiński* AND K. KarpierZ \\ Institute of Experimental Physics, Faculty of Physics, University of Warsaw, Hoża 69, 00-681 Warsaw, Poland \\ Transverse magnetoresistance was studied in monocrystalline GaAs:Te doped above the equilibrium doping \\ limit and annealed to partially deactivate donor impurities. It is shown that in a sample with partial deactivation \\ of Te impurities, which exhibits structural fluctuations in microscale, both strong positive and some negative \\ magnetoresistances arise, which are difficult to understand within the relaxation time approximation in degener- \\ ated homogeneous semiconductor. It is discussed that a consideration of the role of spatial fluctuations (in carrier \\ concentration, conductivity, etc.), e.g. as proposed by Herring, allows for an understanding of positive component \\ of magnetoresistance observed in the sample with a distinct microscopic structural disorder. With the aim to \\ better understand the transport in GaAs:Te, a model material doped above the doping limit, we discuss both \\ positive and negative components of measured magnetoresistance.
}

PACS: 61.72.-y, 61.72.uj, 72.20.My

\section{Introduction}

Semiconductors doped above the equilibrium doping limit show, from a material point of view, features of a continuous phase transition in an alloy, one of which is an onset of structural (critical) fluctuations. For example, in GaAs:Te its microscopic length scale is of the order of micrometer. The electrical equilibrium doping limit considered here may be described as an inherent limitation in ability to high electric doping, e.g. to $n$-type, and it may be defined as the maximum thermodynamically stable concentration of isolated impurities. Semiconducting materials doped above the equilibrium doping limit may show not full electrical activation of impurities, also show a strong sensitivity to thermal annealing [1-5]. The aim of this paper is to better understand the electric transport in very highly doped GaAs:Te, which we treat as a model material above the doping limit.

We compare magnetoresistance for two GaAs:Te highly doped samples, one (denoted below as sample $A$ ) having the concentration of Te impurities approximately equal the equilibrium doping limit, and the other (sample $B$ ) with Te concentration about 4 times higher than the doping limit in GaAs:Te. Preparing sample $B$ an annealing was applied which caused a reduction of the free carrier concentration to a value similar as in sample $A$. This annealing has also caused structural fluctuations in GaAs:Te alloy.

We show that results of transverse magnetoresistance in metallic GaAs:Te with fluctuations above the doping limit go beyond the relaxation time approximation in a

\footnotetext{
* corresponding author; e-mail: tomslu@fuw.edu.pl
}

degenerated homogeneous semiconductor. It is discussed that a description of electric transport in GaAs:Te doped above the doping limit should take into consideration the role of fluctuations e.g. of free carrier concentration, crystal potential etc. related to structural microscopic fluctuations in the alloy.

\section{Samples and experiment}

The Hall bar samples (Table) cut from GaAs:Te single crystals were measured using magnetotransport at fields up to $10 \mathrm{~T}$ and $T=4.2 \mathrm{~K}$. Transverse magnetoresistance (MR) was measured in both directions of magnetic field using DC current of $100 \mathrm{~mA}$. Sample $A$ shows close to full electrical activation of impurities, the free electron concentration $n \approx[\mathrm{Te}]$, where [Te] is the concentration of impurity atoms. Sample $B$ was cut from higher doped GaAs:Te material, which was annealed at $700{ }^{\circ} \mathrm{C}$ resulting in a lowered Hall electron concentration $n<[\mathrm{Te}]$ and structural fluctuations formed in GaAs:Te alloy.

\section{TABLE}

Samples used in studies. Sample $A$ has Te impurity concentration approximately at the equilibrium doping limit. Sample $B$ is doped with Te much above the doping limit. Material of sample $B$ was annealed at $700{ }^{\circ} \mathrm{C}$ for $74 \mathrm{~h}$ to reduce the free carrier concentration to $n=3.4 \times 10^{18} \mathrm{~cm}^{-3}$.

\begin{tabular}{c|c|c}
\hline \hline Sample & $\begin{array}{c}\text { Te concentration } \\
{\left[\mathrm{cm}^{-3}\right]}\end{array}$ & $\begin{array}{c}\text { Hall concentr. } \\
{\left[\mathrm{cm}^{-3}\right]}\end{array}$ \\
\hline$A, \#$ B43RA15 & $4 \times 10^{18}$ & $3.5 \times 10^{18}$ \\
$B, \#$ B42LA2 & $1.7 \times 10^{19}$ & $3.4 \times 10^{18}$
\end{tabular}




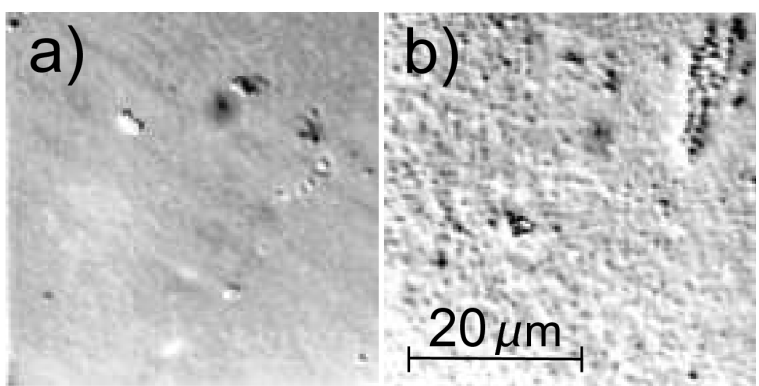

Fig. 1. Optical metallographic microscopy pictures of polished and then selectively etched GaAs:Te (100) surface. (a) Sample with almost complete electrical activation $n \approx[\mathrm{Te}]$, like sample $A$. (b) Highly doped sample $\left([\mathrm{Te}]=1.5 \times 10^{19} \mathrm{~cm}^{-3}\right)$ with concentration of free electrons reduced by annealing at $740{ }^{\circ} \mathrm{C}$ for $92 \mathrm{~h}$, like sample $B$. Much stronger structural fluctuations are visible in sample $B$.

Figure 1 shows optical metallographic microscopy pictures of GaAs:Te samples mirror-polished and then chemically etched in a selective etchant. Samples with free-electron concentration reduced from $[\mathrm{Te}]=1.5 \times$ $10^{19} \mathrm{~cm}^{-3}$ to $n \approx 4 \times 10^{18} \mathrm{~cm}^{-3}$ (having part of Te impurity deactivated) exhibit distinct structural fluctuations - Fig. 1b. We interpret these fluctuations as regions containing fluctuating concentrations of few-impurity aggregates like $\mathrm{Te}-\mathrm{Te}$ or $\mathrm{Te}-\mathrm{Te}-\mathrm{Te}$ etc., having locally reduced concentration of free electrons and being strained $[5,6]$.

\section{Results and discussion}

In Fig. 2 the results of symmetric part $(B+, B-)$ of measured resistance, $\operatorname{MR}(B) \equiv\left[\rho_{x x}(B)-\rho_{x x}(0)\right] / \rho_{x x}(0)$, are shown. Sample $A$ shows relatively small MR up to fields about $6 \mathrm{~T}$ which is consistent with close to zero MR expected within the relaxation time approximation in a homogeneous metallic semiconductor. In strongly degenerated homogeneous semiconductors $\operatorname{MR}(B)=$ $(\mu B)^{2}\left(\left\langle\tau_{\mathrm{m}}^{3}\right\rangle\left\langle\tau_{\mathrm{m}}\right\rangle-\left\langle\tau_{\mathrm{m}}^{2}\right\rangle^{2}\right) /\left\langle\tau_{\mathrm{m}}\right\rangle^{4}$ should be zero due to the energy averaged momentum relaxation time $\left\langle\tau_{\mathrm{m}}^{n}\right\rangle=$ $\left(\tau\left(E_{\mathrm{F}}\right)\right)^{n}$, where $E_{\mathrm{F}}$ is the Fermi energy, $n=1,2, \ldots$ The Shubnikov-de Haas $(\mathrm{SdH})$ oscillations visible for $B>7 \mathrm{~T}$ confirm metallic character of conductivity and give the Fermi energy $E_{\mathrm{F}}=99 \mathrm{meV}$, Ref. [7] (p. 303), which is consistent within $20 \%$ with the value expected for $n=3.5 \times 10^{18} \mathrm{~cm}^{-3}$.

MR results for sample $B$ are significantly different in spite of similar concentrations of free electrons in both samples $A$ and $B$. MR (about $3 \%$ at $B=10 \mathrm{~T}$ ) is larger than in sample $A$, and has clearly visible positive component above $\approx 1 \mathrm{~T}$ and negative one below. So strong positive MR cannot be well understood assuming degenerated homogeneous semiconductor and relaxation time picture mentioned above. The most obvious difference between samples $A$ and $B$ seems to be related with various types of defects responsible for a lowered electrical activation of donors in sample $B$, including structural

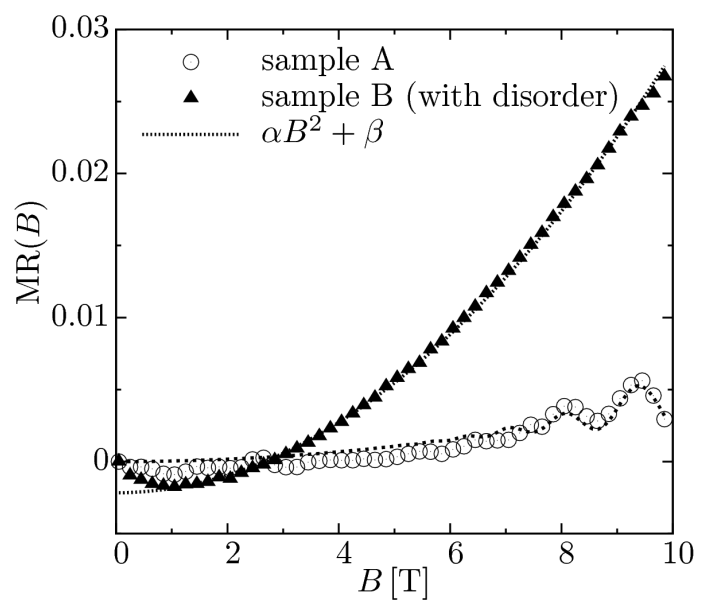

Fig. 2. Symmetric part $(B+, B-)$ of magnetoresistance measured in the Hall bar samples $A$ and $B$. In sample $A \mathrm{SdH}$ oscillations are clearly visible and fitted. In sample $B$ the positive component of MR can be fitted with a quadratic dependence.

fluctuations, created during annealing. We assume that the defects containing inactive Te impurities, presumably Te-Te clusters etc., are not distributed uniformly in a crystal, but their concentration fluctuates with the characteristic length scales of about $1 \mu \mathrm{m}$. This distribution is responsible for structural fluctuations visible in Fig. $1 \mathrm{~b}$ and also for the local fluctuations of carrier concentration. Looking for a proper description of MR observed, we consider, following Herring [8], that spatial fluctuations of carrier concentration or conductivity in a sample result in a positive quadratic MR. It was shown by Herring that the Fourier components of fluctuating conductivity do contribute to the effective conductivity tensor components and thus influence measured effective resistivity, $\operatorname{MR}(B) \propto\left(\mu_{\mathrm{H}} B\right)^{2}(\Delta n /\langle n\rangle)^{2}$, where $\Delta n$ is an amplitude of electron concentration's fluctuation, $\langle n\rangle$ is spatial average of electron concentration, and $\mu_{\mathrm{H}}$ is the Hall mobility assumed here as spatially uniform (which, however, is rather not the case of sample $B$ with spatial fluctuations of ionized donor concentration and local strain due to Te-Te aggregates). The fluctuations of carrier concentration do not alter the interpretation of the Hall coefficient $R=1 /\langle n\rangle e$ under similar assumptions. Based on this picture, the fluctuations of electron concentration $\Delta n$ in sample $B$ could be estimated as $\Delta n \approx 0.7\langle n\rangle$.

The characteristic length scale of fluctuations in sample $B$ is about $1 \mu \mathrm{m}$, as it follows from metallographic (Fig. 1) or X-ray studies $[5,6]$. It should be noticed that the fluctuation size is much larger than the electron wavelength at the Fermi level $\lambda_{\mathrm{F}} \approx 15 \mathrm{~nm}$ and the mean free path $\ell=\tau_{\mathrm{m}} v_{\mathrm{F}} \approx(14 \div 30) \mathrm{nm}$, where $\tau_{\mathrm{m}}$ is momentum relaxation time, $\tau_{\mathrm{m}} \approx 15$ fs from the Hall mobility $\mu_{\mathrm{H}} \approx 500 \mathrm{~cm}^{2} /(\mathrm{Vs})$ or $\tau_{\mathrm{m}} \approx 40$ fs from the Shubnikov-de Haas oscillations in sample $B$, and $v_{\mathrm{F}}$ is the velocity at the Fermi level. Consequently, the classi- 
cal picture of transport in slowly fluctuating potential, as used in [8], seems appropriate in a case of sample $B$. Measurements for sample $B$ are mostly in the low field limit since $\mu_{\mathrm{H}} B \approx 0.5$ for $B=10 \mathrm{~T}$ and $\mu_{\mathrm{H}} \approx 500 \mathrm{~cm}^{2} /(\mathrm{V} \mathrm{s})$ at $T=4.2 \mathrm{~K}$.

In Fig. 3 we show the components of MR for sample $B$ obtained after subtracting the quadratic dependence $\propto B^{2}$ shown in Fig. 2. We may clearly see a negative component of $\mathrm{MR}$ for $B<2 \mathrm{~T}$ and also the Shubnikov-de Haas oscillations at fields $B=(7 \div 10) \mathrm{T}$. $\mathrm{SdH}$ oscillations confirm metallic type of transport in sample $B$, similar like in sample $A$, and the fit with $\mathrm{SdH}$ dependence yields the Fermi energy about $110 \mathrm{meV}$, which is well consistent with measured Hall concentration $n \approx 3.4 \times 10^{18} \mathrm{~cm}^{-3}$, as well as the relaxation time $\tau_{\mathrm{m}} \approx 40 \mathrm{fs}$, as mentioned above.

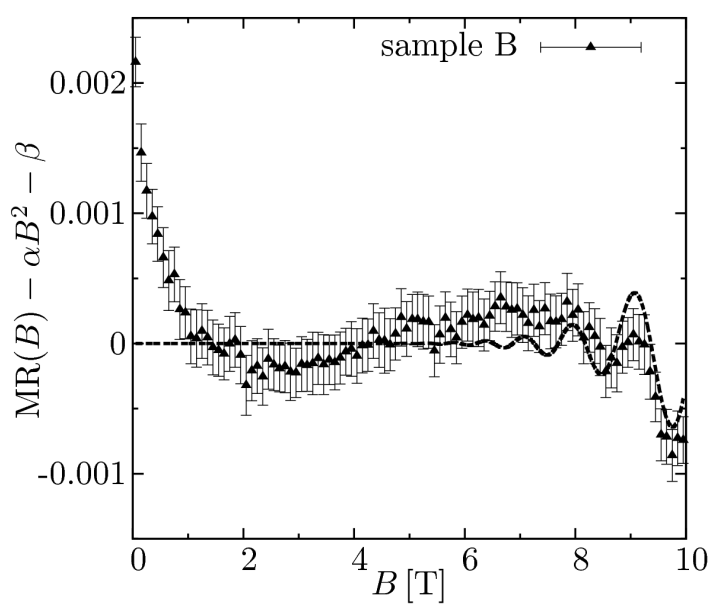

Fig. 3. Remnant part of MR in sample $B$ after subtracting the quadratic dependence. Negative component of MR and SdH oscillations are visible.

Interesting, however more difficult to understand, is the negative component of MR in sample $B$ observed for $B<2 \mathrm{~T}$. MR in this region seems to decay exponentially like $\mathrm{e}^{-B / B_{0}}$. We observe negative $\mathrm{MR}$ when the cyclotron radius $R_{\mathrm{c}}$ is of the size of potential fluctuations correlation length $d \approx 1 \mu \mathrm{m}$, e.g. $R_{\mathrm{c}} \approx 1000 \mathrm{~nm}$ for $B \approx 0.3 \mathrm{~T}$. We may suppose that the negative part is also related to the fluctuations present in the sample $B$ and search for a "classical" origin of negative MR. The possible origins of negative MR may be related to a classical "memory effect" in transport, as considered recently in 2D electron gas [9-11], related to a deviation from the relaxation time approximation, in our case enhanced in some way by the defects formed at the doping limit or by the macroscopic critical fluctuations. On the other hand, effects of quantum interference resulting in the weak localization and destroyed by a magnetic field seem to fit fairly well the negative part of MR observed in sample $B$ [12]. Since, principally, the weak localization occurs in the regime of phase-coherence of electron wave, a relatively short mean free path $\ell \approx(14 \div 30) \mathrm{nm}$ in sample $B$, comparable with the reciprocal Fermi wavevector $\ell \approx 1 / k_{\mathrm{F}}$, seems not to destroy the electron coherence.

\section{Acknowledgments}

Authors are grateful for colleagues at University of Warsaw who assisted in the experiments, K. Nogajewski, M. Białek and Dr. J. Borysiuk. Work was supported in part by MTKD-CD-2005-029671 research framework.

\section{References}

[1] C.S. Fuller, K. Wolfstirn, J. Appl. Phys. 34, 2287 (1963).

[2] J.K. Kung, W.G. Spitzer, J. Appl. Phys. 44, 912 (1973).

[3] J.K. Kung, W.G. Spitzer, J. Appl. Phys. 45, 4477 (1974).

[4] M.G. Milvidskii, W.B. Osvenskii, V.I. Fistul, E.M. Omeljanovskii, S.P. Grishina, Fiz. Tekh. Poluprovodn. 1, 969 (1967).

[5] T. Słupiński, Ph.D. Thesis, University of Warsaw, Warsaw 1999.

[6] T. Słupiński, E. Zielińska-Rohozińska, in: Mater. Res. Soc. Symp. Proc., Eds. A. Mascarenhas, D. Follstaedt, T. Suzuki, B. Joyce, Materials Research Society, Warrendale, Pennsylvania 2000, p. 261.

[7] K. Seeger, Semiconductor Physics. An Introduction, 7 ed., Springer-Verlag, Berlin 1999.

[8] C. Herring, J. Appl. Phys. 31, 1939 (1960).

[9] D.G. Polyakov, F. Evers, A.D. Mirlin, P. Wölfle, Phys. Rev. B 64, 205306 (2001).

[10] A.D. Mirlin, D.G. Polyakov, F. Evers, P. Wölfle, Phys. Rev. Lett. 87, 126805 (2001).

[11] A.P. Dmitriev, V.Yu. Kachorovskii, Semiconductors 42, 934 (2008) [Fiz. Tekh. Poluprovodn. 42, 950 (2008)].

[12] A. Kawabata, Solid State Commun. 34, 431 (1980). 